OPEN ACCESS

Edited by:

Se-Young Choi,

Seoul National University,

South Korea

Reviewed by:

Hiroshi Kuba,

Nagoya University, Japan

Matthew Van Hook

University of Nebraska Medical

Center, United States

${ }^{*}$ Correspondence:

Jun Hee Kim

kimjh@uthscsa.edu

Specialty section:

This article was submitted to

Cellular Neurophysiology,

a section of the journa

Frontiers in Cellular Neuroscience

Received: 31 December 2020

Accepted: 09 March 2021

Published: 09 April 2021

Citation:

Kim EJ, Nip K, Blanco C and Kim JH (2021) Structural Refinement of the Auditory Brainstem Neurons

in Baboons During Perinatal

Development.

Front. Cell. Neurosci. 15:648562. doi: 10.3389/fncel.2021.648562

\section{Structural Refinement of the Auditory Brainstem Neurons in Baboons During Perinatal Development}

\author{
Eun Jung Kim¹, Kaila Nip ${ }^{1}$, Cynthia Blanco ${ }^{2}$ and Jun Hee Kim ${ }^{1 *}$ \\ ${ }^{1}$ Department of Cellular and Integrative Physiology, University of Texas Health Science Center, San Antonio, TX, \\ United States, ${ }^{2}$ Department of Pediatrics, University of Texas Health Science Center, San Antonio, TX, United States
}

Children born prematurely suffer from learning disabilities and exhibit reading, speech, and cognitive difficulties, which are associated with an auditory processing disorder. However, it is unknown whether gestational age at delivery and the unnatural auditory environment in neonatal intensive care units (NICU) collectively affect proper auditory development and neuronal circuitry in premature newborns. We morphologically characterized fetal development of the medial superior olivary nucleus (MSO), an area important for binaural hearing and sound localization, in the auditory brainstem of baboon neonates at different gestational ages. Axonal and synaptic structures and the tonotopic differentiation of ion channels in the MSO underwent profound refinements after hearing onset in the uterus. These developmental refinements of the MSO were significantly altered in preterm baboon neonates in the NICU. Thus, the maternal environment in uterus is critical for auditory nervous system development during the last trimester of pregnancy and critically affects the anatomic and functional formation of synapses and neural circuitry in the preterm newborn brain.

Keywords: non-human primate, auditory brainstem, MSO, fetal development, NICU, structural plasticity, synapse, axon initial segment (AIS)

\section{INTRODUCTION}

Prematurity is one of the leading causes of perinatal mortality and long-term disability. Premature infants are at high risk for motor, sensory, cognitive, and behavioral disabilities (Fanaroff et al., 2007; Griffith et al., 2012). In particular, neurosensory impairments such as deafness and visual deficits occur in 5-15\% of preterm newborns (Johnson et al., 2005; Wilson-Costello et al., 2005; Moster et al., 2008). Auditory impairments caused by premature birth can profoundly affect reading and speaking ability and the development of language, all of which are associated with auditory processing disorder due to the inefficiency or incapacity of the auditory system to process acoustic information (Moore, 2002; McClure et al., 2005). Preterm birth significantly increases the risk of neurodevelopmental disorders, such as autism spectrum disorder, that are characterized by some degree of auditory dysfunction including deafness, hyperacusis, difficulty listening in the presence of background noise, and problems in orienting and encoding speech sounds (O'Connor, 2012; Agrawal et al., 2018). Notably, binaural hearing plays a key role in the development of speech and language perception because the normal development of the brain's binaural circuitry requires the proper processing of auditory information (Lieu et al., 2010; Franken et al., 2014). For instance, a clinical study showed a delay in sound localization at 12 months was associated with deficits in the 
physiologic mechanism of temporal processing at an auditory processing evaluation done between 4 and 7 years of age (Gallo et al., 2011). Thus, binaural hearing, sound localization, and temporal processing require more extensive central processing, the development of which is influenced by auditory experiences in different species from rodents to primates (Friauf and Lohmann, 1999; Moore, 2002).

The medial superior olivary nucleus (MSO) is an auditory brainstem nucleus and an important binaural site in the auditory pathway, where auditory inputs from the two ears converge and calculate the interaural time differences for computing sound location (Joris et al., 1998). The MSO is a prominent nucleus found in most small mammalian species. The biophysical, morphologic, synaptic, and biochemical properties of MSO neurons have been well described in gerbils, guinea pigs, mice, rats, and bats during their postnatal development (Kapfer et al., 2002; Grothe and Pecka, 2014; Fischl et al., 2016). However, there are drastic differences in the developmental time-course and initiation factors of myelination and synaptic innervation between primates and rodents (Moore and Linthicum, 2007; McMahon et al., 2012; Miller et al., 2013). Unlike mice and rats, human fetuses already have substantial capacity for auditory learning and memory (Moore, 2002; Moore and Linthicum, 2007; Gallo et al., 2011), as they start to hear and undergo major developmental changes in the auditory system during the third trimester of pregnancy in the uterus (Moore and Linthicum, 2007). Understanding the neurophysiological effects of auditory experience on the MSO in the developing brain of non-human primate is essential to comprehend how experience-dependent plasticity drives the development of auditory processing in human.

Here, we investigated morphologic profiles of MSO neurons, including axonal structure, synapse, and tonotopic organization of ion channels throughout fetal development, specifically before and after hearing onset, in baboon neonates delivered at different gestational ages. Baboon fetuses begin to hear in the uterus at $\sim 70 \%$ of gestational age (GA) and display five distinguishable waves of auditory brainstem responses (ABRs) at $\sim 80 \%$ of GA (Edwards et al., 1994), similar to humans. Baboons preferentially respond to lower-frequency sounds $(<8 \mathrm{kHz}$, threshold: $\sim 40 \mathrm{~dB}$ ), as do human newborns. Studying the baboon brain thus allows direct comparisons with the human brain because the connectivity, size, and functional areas (reflected in motor and behavioral capacities) are highly similar to those in humans. Premature baboons delivered at 126 days (d) GA (term $=180$ days GA), share a similar neonatal course with human preterm infants delivered at 28 weeks of GA (extremely preterm), with common complications relevant to prematurity such as incomplete lung development and metabolism (Blanco et al., 2013).

In addition, we studied how the altered auditory environment immediately after hearing onset influences the morphologic features of the MSO during early development. Preterm newborns experience a drastic change in going from the dark and muffled environment of the uterus to the unnatural environment of the NICU. During NICU stays, gestational age, medications, procedures, the unnatural auditory environment, and other stimuli can cause significant stress associated with adverse auditory development in preterm newborns (McMahon et al., 2012). A difference of auditory environment between the maternal womb and the NICU, for instance attenuated maternal sound (e.g., maternal heart-beat sound) and increased unnecessary noise produced by ventilators, infusion pumps, or alarms, can cause potential risk for compromised neuroplasticity of the auditory nervous system in preterm newborns. We studied how shorten time in uterus and altered auditory environment in the NICU impact the structural development and refinement of the MSO at the cellular level in developing baboon brains. These studies provide the first systematic description of MSO development at the cellular and synaptic level in non-human primates and reveals how premature birth alters the normal development of the MSO in the auditory brainstem.

\section{RESULTS}

\section{Morphologic Profiles of the MSO in Baboon Neonates During Fetal Development}

Similar to the superior olivary complex (SOC) of humans (Kulesza and Mangunay, 2008; Kulesza and Grothe, 2015), the SOC from the baboon neonate brainstem delivered at 140d GA after hearing onset consists of the medial nucleus of the trapezoid body (MNTB), the MSO, the lateral superior olivary nucleus (LSO), the ventral nucleus of the trapezoid body (VNTB), and the lateral nucleus of the trapezoid body (LNTB). Baboon auditory brainstems labeled with MAP2 show prominent MNTB, MSO, and LSO organized in the medial to lateral directions in consecutive order. The MNTB was the most medial nucleus, with a round shape located along the auditory fibers that cross the midline of the brainstem (Kim et al., 2014). The MSO, a dorsoventrally orientated column of neurons, was located laterally to the MNTB. MAP2 staining showed MSO neurons with bipolar-like dendrites extending toward both the medial MNTB and lateral LSO. In the perinatal stage at 140d $\mathrm{GA}$, individual neurons from those prominent nuclei (MNTB, MSO, and LSO) already had a typical morphology (e.g., bipolar structure of MSO neurons or multiple dendrites from LSO) and firing patterns (Figures 1A-C). In whole-cell recordings of MSO neurons from preterm baboon neonates (extremely or moderate preterm baboons; hereafter E-Pre or M-Pre, respectively), a prolonged depolarizing current injection (200 ms) resulted in a single action potential in a phasic manner, which is a common feature of MSO neurons (Khurana et al., 2011; Fischl et al., 2016). A phasic plotting the $\mathrm{dV} / \mathrm{dt}$ of action potential showed the MSO neurons have a relatively high threshold $(-28.0 \pm 3.96 \mathrm{mV}, n=5$ cells for 3 E-Pre baboons and $-26.8 \pm 4.37 \mathrm{mV}, n=4$ cells for 3 M-Pre baboons) and a lower amplitude of action potentials, measured by voltage change from the threshold to the peak potential $(71.9 \pm 3.311 \mathrm{mV}, n=5$ cells for E-Pre and $74.1 \pm 3.53$, $n=4$ cells for M-Pre, Figure 1D). Action potential width, measured by the time distance between the peaks in the $\mathrm{dV} / \mathrm{dt}$, was $0.36 \pm 0.009 \mathrm{~ms}(n=5$ cells $)$ at $67 \% \mathrm{GA}$ and $0.38 \pm 0.061 \mathrm{~ms}$ ( $n=4$ cells, data not shown). The input resistance was $115 \pm 18.6$ 


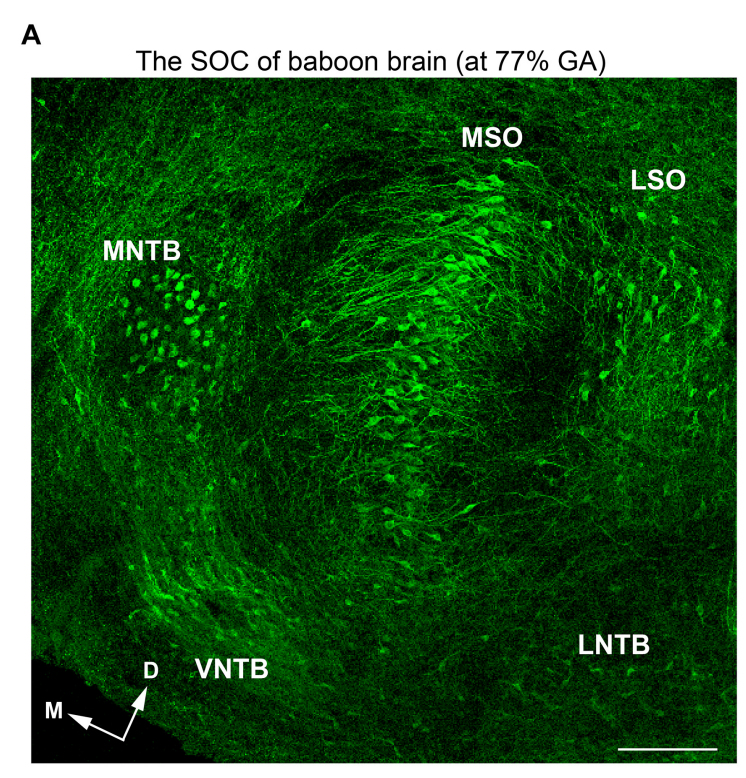

B

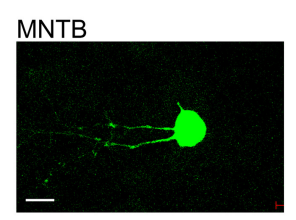

MSO

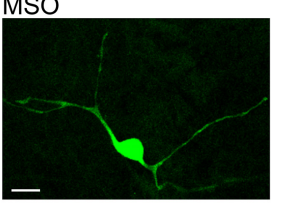

LSO

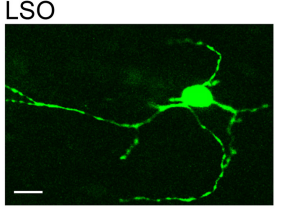

C
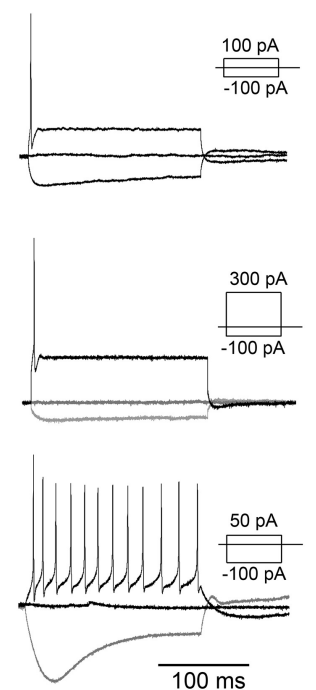

D
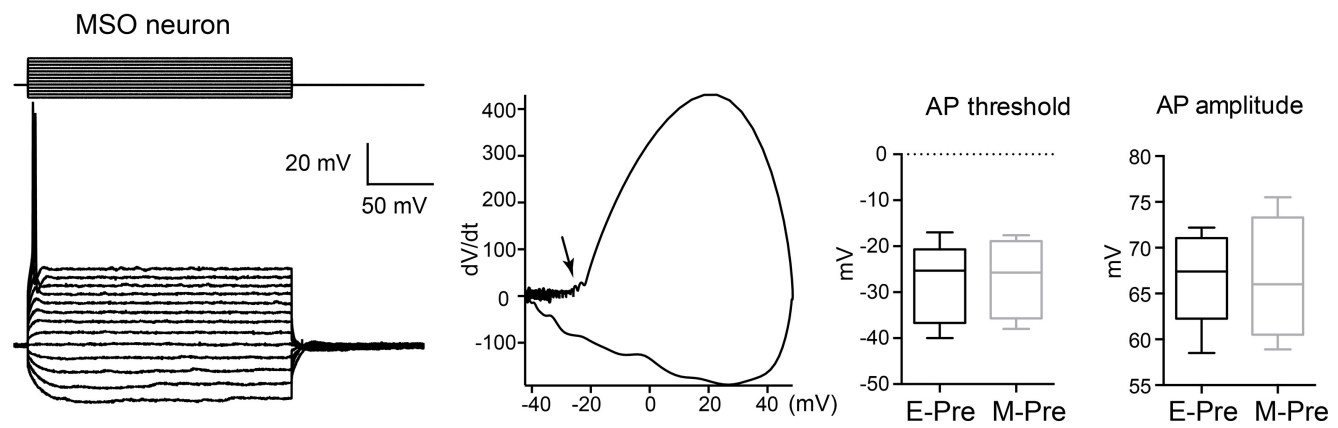

FIGURE 1 | The main nuclei of the baboon SOC. (A) The SOC in the auditory brainstem from a baboon neonate delivered at $77 \%$ GA (moderate preterm; M-Pre), immunolabeled with MAP2 (green). From the midline of the brainstem, the MNTB, MSO, and LSO were arranged in a lateral direction. Scale $=200 \mu \mathrm{m}$. (B) Individual cells from LSO, MNTB, and MSO nuclei were filled with Alexa dye during whole-cell recordings. Scale $=50 \mu \mathrm{m}$. (C) Representative trace of membrane potentials of individual neurons. Action potentials from the same cells in B were evoked by threshold current injections. Inset, depolarizing and hyperpolarizing current injections. (D) Representative trace of membrane potentials of MSO neuron from a M-Pre neonate in response to prolonged current injection (from -200 pA to 400 pA, $\triangle 50$ pA for $200 \mathrm{~ms}$ ) at holding $-70 \mathrm{mV}$. (Middle) Phasic plotting of a single AP (dV/dt against membrane voltage) triggered by current injection (300 pA). Arrow indicates the AP threshold. (Right) The AP threshold and amplitude of MSO neurons from extreme preterm (E-Pre) and M-Pre baboon neonates. In box and whisker plot, boxes indicate $25-75 \%$ interquartile range and horizontal lines in boxes indicate median.

$\mathrm{M} \Omega(n=4$ cells $)$ for E-Pre and $97 \pm 12.6 \mathrm{M} \Omega(n=4)$ for M-Pre. The membrane time constant was $5.9 \pm 1.66 \mathrm{~ms}$ ( $n=3$ cells) for E-Pre and $3.9 \pm 1.28 \mathrm{~ms}$ ( $n=4$ cells, data not shown) for M-Pre. Both had a decreasing trend during gestational development, but showed no significant differences between E-Pre and M-Pre baboon neonates.

To determine the effects of auditory experience in the uterus on MSO development after hearing onset, we compared morphologic changes of the MSO in baboon neonates delivered at different gestational ages - E-Pre, M-Pre, and full-term birth; these corresponded to $67 \%, 77 \%$, and $100 \%$ of $180-185$ gestational days (full-term neonate, Figure 2A). Most MSO neurons labeled with MAP2 exhibited a bipolar structure of primary dendrites that protruded toward both the medial MNTB and lateral LSO (Figure 2B). Overall, the MSO became longer and narrower, transforming the outer line of MSO nuclei from an oval to a column shape with ventral-dorsal orientation during fetal development. We analyzed the MSO shape and area using draw spline contour tool to outline around the outside of the bipolar extensions of the MAP2 + cluster of MSO neurons. In E-Pre baboons before hearing onset, the MSO was an oval shape $\left(306,331 \pm 22,794 \mu \mathrm{m}^{2}, n=7\right.$ slices from 5 E-Pre baboons) and aligned along the ventral-dorsal axis. After hearing onset in M-Pre baboons, the outer nuclei shape of the MSO highlighted by MAP2 + neurons became more condensed and narrower $\left(283,045 \pm 29,823 \mu \mathrm{m}^{2}, n=7\right.$ slices from 5 M-Pre baboons vs $306,331 \pm 22,794 \mu \mathrm{m}^{2}, n=7$ slices from 5 E-Pre baboons). In fullterm baboons (hereafter Term), the MSO was much thinner and longer than in preterm baboons $\left(345,652 \pm 21,317 \mu \mathrm{m}^{2}, n=11\right.$ slices from 3 baboons). MSO area per tissue was not significantly different in the gestational development groups, but the MSO neuron alignment along the dorsal-ventral axis was significantly 


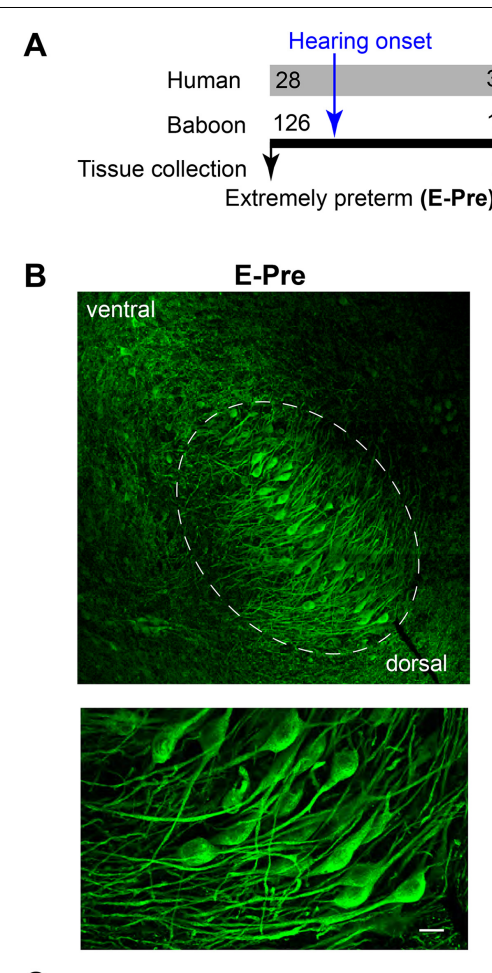

C
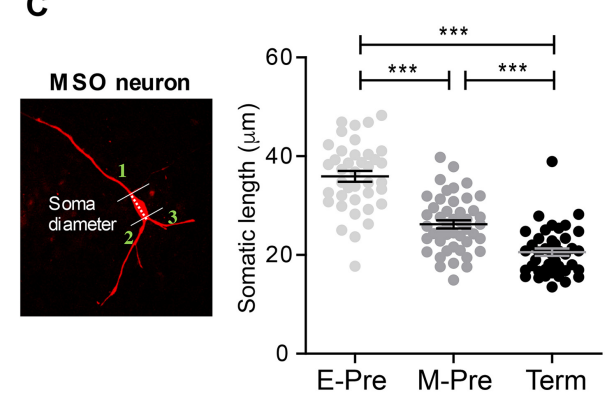
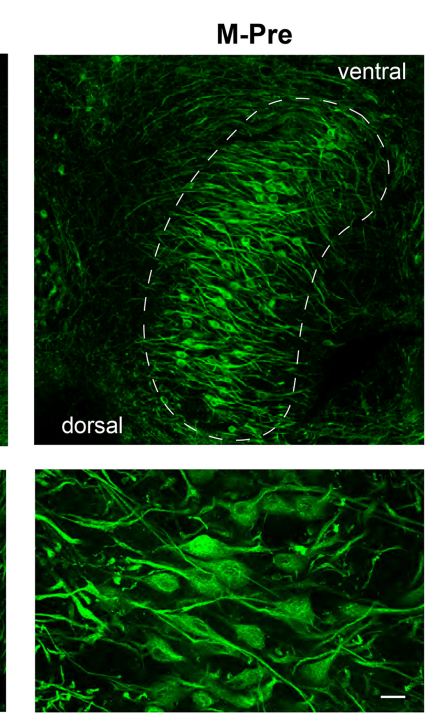

D
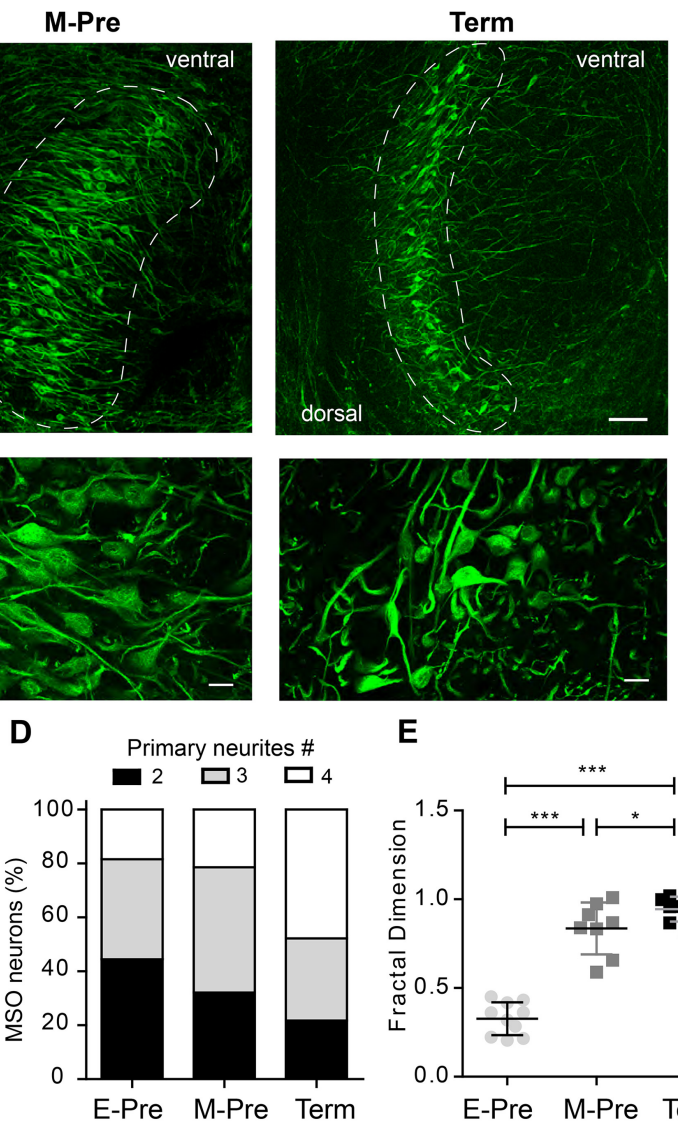

E

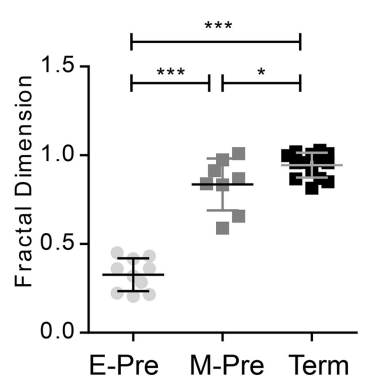

FIGURE 2 | Morphologic changes in MSO neurons during gestational development of baboons in the uterus. (A) Timeline for the last trimester of human (weeks) and baboon (days of gestational age, GA) gestation periods. Hearing onset is around 28 weeks of GA for human fetuses and after 126 days GA for baboons (blue arrow). Auditory brainstem tissues were collected from baboon neonates delivered at 126d GA (E-Pre) and 140d GA (M-Pre) via cesarean section, and full-term (Term) through natural delivery. Black arrows indicate the tissue collection time. (B) MSO neurons were immunostained with MAP2 in the auditory brainstem from E-Pre, M-Pre, and Term neonates. (Top) Circles indicate the MSO area. Scale $=100 \mu \mathrm{m}$. (Bottom) Magnified image of MSO neurons. Scale $=20 \mu \mathrm{m}$. (C) A single MSO neuron from a M-Pre neonate filled by Alexa dye during whole-cell recording. Broken line indicates the somatic length and numbers indicate primary dendrites extending from the soma counted for quantitative analysis. Right: Somatic length from MSO neurons at different GA. (D) Proportions of primary dendrites in MSO neurons at different GA. (E) The fractal dimension, a measure of dendritic complexity, increased throughout the gestational period in E-Pre, M-Pre, and Term neonates. Data were shown as mean \pm s.e.m. ${ }^{*}<0.05,{ }^{\star *}<0.01$, and ${ }^{* *}<0.001$. One-way ANOVA and post hoc Tukey's multiple comparison test.

longer in Term baboons ( $1,074 \pm 57.2 \mu \mathrm{m}, n=8$ slices $)$ compared to E-pre (765 $\pm 62.4 \mu \mathrm{m}, n=11$ slices, $p=0.0025$, MannWhitney test). The long axes of MSO neurons were arranged in a geometrically precise orientation with a dorsal to ventral direction (Figure 2B). These results reveal a developmental change in the shape of the MSO during the last trimester of pregnancy in baboon brains.

Next, we analyzed morphologic parameters of individual MSO neurons from preterm and full-term baboon neonates. The soma diameters of MSO neurons were $35.93 \pm 0.101 \mu \mathrm{m}(n=39$ cells) in 4 E-Pre baboons, $26.22 \pm 0.819 \mu \mathrm{m}$ ( $n=46$ cells $)$ in 4 M-Pre baboons, and $20.57 \pm 0.758 \mu \mathrm{m}(n=41$ cells $)$ in 3
Term baboons ( $p=0.0005$ by nested one-way ANOVA followed by Tukey's multiple comparison test, Figure 2C). In E-Pre neonates, $\sim 50 \%$ of MSO neurons had two primary dendrites, showing a clear bipolar structure. In M-Pre neonates, $\sim 70 \%$ of MSO neurons had more than three primary dendrites extending from the soma. In Term neonates, $\sim 80 \%$ of MSO neurons had multiple primary dendrites (Figure 2D). MSO neurons analyzed using theoretical fractal dimension analysis (Karperien et al., 2013) were significantly more complex in M-Pre baboon neonates $(0.83 \pm 0.051, n=8$ cells) after hearing onset and further increased in Term neonates ( $0.94 \pm 0.0185, n=14$ cells) compared to E-Pre baboons before hearing onset $(0.32 \pm 0.029$, 
$n=10$ cells, $\mathrm{p}<0.0001$ by one-way ANOVA followed by Tukey's multiple comparison test, Figure 2E). Taken together, these observations show that the MSO underwent significant morphologic changes in the outer shape of the nucleus, soma size, and the complexity of dendritic arbors in neurons after hearing onset in the uterus.

\section{Excitatory and Inhibitory Inputs on MSO Neurons After Hearing Onset During Fetal Development in the Uterus}

Morphologic development of MSO neurons, including dendritic structure, may be regulated by synaptic inputs and integration (Deitch and Rubel, 1984; Chirila et al., 2007). We previously demonstrated that spatial refinement of synaptic inputs on the dendritic and somatic surfaces of the MSO neuron from baboon neonates occur after hearing onset (Kim et al., 2014). In the MSO of extremely preterm baboons, the vesicular GABA transporter (VGAT), which is a marker for inhibitory terminals, was visible on both cell bodies and dendrites; in term baboons, the MSO VGAT and vesicular glutamate transporter 1 (VGluT1, a marker for excitatory nerve terminals) puncta were concentrated on the soma and dendrites of MSO neurons, respectively (Figures 3A,B). Immunostaining of VGluT1 and VGAT showed that individual VGluT1 puncta area significantly increased in M-Pre MSO after hearing onset compared to E-Pre neonates $\left(1.38 \pm 0.041 \mu \mathrm{m}^{2}, n=193\right.$ puncta from 6 slices in 2 E-Pre baboons, $2.69 \pm 0.142 \mu \mathrm{m}^{2}, n=159$ puncta from 6 slices in 2 M-Pre baboons, and $4.05 \pm 0.084 \mu \mathrm{m}^{2}, n=425$ puncta from 5 slices in 2 Term baboons, $p=0.0005$ by nested oneway ANOVA, Figure 3C). VGAT puncta area also increased $\left(1.03 \pm 0.034 \mu \mathrm{m}^{2}, n=196\right.$ puncta in E-Pre, $2.14 \pm 0.152 \mu \mathrm{m}^{2}$, $n=145$ puncta in M-Pre, and $3.02 \pm 0.125 \mu \mathrm{m}^{2}, n=220$ puncta in Term, $p=0.002$ by nested one-way ANOVA, Figure 3D). The number of VGluT1 puncta within a defined area $\left(1000 \mu \mathrm{m}^{2}\right)$ was $30.45 \pm 4.765$ ( $n=4$ slices) in E-Pre, $15.97 \pm 3.851(n=5$ slices) in M-Pre, and $13.75 \pm 2.087(n=6$ slices $)$ in Term ( $p=0.0134$, one-way ANOVA followed by Tukey's multiple comparison test). The number of VGAT puncta within a defined area $\left(1000 \mu \mathrm{m}^{2}\right)$ of MSO was $43.13 \pm 6.936(n=4$ slices $)$ in E-Pre, $12.25 \pm 2.398$ ( $n=5$ slices $)$ in M-Pre, and $11.81 \pm 0.988$ $(n=9$ slices $)$ in Term $(p<0.0001$, one-way ANOVA followed by Tukey's multiple comparison test, Figure 3E). The number of VGluT1 and VGAT puncta significantly decreased after hearing onset (between E-Pre and M-Pre) and remained this reduction throughout Term neonates. The increase in the size of VGluT1 and VGAT puncta could be due to changes in total vesicle number within individual boutons or distribution of synaptic vesicles around the active zone.

Ultrastructural analysis of synapses in the MSO using transmission electron microscopy (TEM) showed no significant differences in the average active zone length $(377.1 \pm 17.68 \mathrm{~nm}$, $n=37$ for E-Pre, $399.4 \pm 18.29 \mathrm{~nm}, n=42$ for M-Pre, and $436 \pm 27.0 \mathrm{~nm}, n=20$ for Term, $p=0.175$, one-way ANOVA and post hoc Tukey's multiple comparison, Figures $4 \mathbf{A}, \mathbf{B})$. However, the vesicles around the active zone (within $200 \mathrm{~nm}$ from the plasma membrane) and docked vesicles increased in Term neonates. Docked vesicles (within $10 \mathrm{~nm}$ ) were $1.47 \pm 0.24, n=36$ for E-Pre, $1.98 \pm 0.25, n=42$ for M-Pre, and $3.0 \pm 0.37, n=18$ for Term ( $p=0.0052$, one-way ANOVA and post hoc Tukey's multiple comparison, Figure 4C). The numbers of vesicles located within $100 \mathrm{~nm}(6.29 \pm 0.68, n=37$ for E-Pre, $6.33 \pm 0.48$, $n=42$ for M-Pre, or $10.5 \pm 1.10, n=18$ for Term) and $200 \mathrm{~nm}$ $(11.58 \pm 1.22, n=36$ for E-Pre, $12.95 \pm 0.88, \mathrm{n}=42$ for M-Pre, or $19.0 \pm 1.83, n=18$ for Term) were also increased throughout the gestational development (one-way ANOVA and Tukey's multiple comparison, Figure 4C). In particular, the number of docked vesicles per the defined active zone ( $100 \mathrm{~nm}$ length, $0.34 \pm 0.056$, $n=41$ for E-Pre vs $0.82 \pm 0.058, n=30$ for Term, $p<0.001$, unpaired $t$-test) and total vesicle numbers $(29.1 \pm 3.41, n=34$ for E-Pre vs $60.3 \pm 5.92, n=18$ for Term, $p<0.001$, unpaired $t$-test) were significantly increased in Term neonates compared with E-Pre neonates (Figure 4D). These results indicate that the distribution and strength of excitatory and inhibitory synaptic inputs to the MSO are dynamically refined by auditory inputs during the period of fetal development occurring after hearing onset in the uterus.

\section{Developmental Refinement of $\mathrm{K}^{+}$ Channel Expression in the MSO Neurons in the Uterus}

To determine whether developmental changes in synaptic and dendritic structures are accompanied with ion channel expression of MSO neurons, we examined $\mathrm{K}^{+}$channel expression in MSO neurons. MSO neurons in gerbils, rats, and mice exhibit low- and high-voltage activated potassium $\left(\mathrm{K}_{\mathrm{v}}\right)$ channels, which critically contribute to action potential firing in MSO neurons (Scott, 2005; Chirila et al., 2007; Fischl et al., 2016; Nabel et al., 2019). We examined expression of low-voltageactivated $\mathrm{K}_{\mathrm{v}} 1.2$ and high-voltage-activated $\mathrm{K}_{\mathrm{v}} 3.1$ channel in MSO neurons during gestational development. In M-Pre neonates, $\mathrm{K}_{\mathrm{v}} 3.1$ channel was expressed in the somatic and primary dendritic membranes of MSO neurons, whereas $\mathrm{K}_{\mathrm{v}} 1.2$ channel expression was restricted to the proximal axons of MSO neurons (Figure 5A). $\mathrm{K}_{\mathrm{v}} 3.1$ channel was broadly expressed in the somatic and primary dendritic membranes of MSO neurons in E-Pre throughout Term baboons, whereas $\mathrm{K}_{\mathrm{v}} 1.2$ channel expression underwent a developmental refinement. Notably, the length of the observed $\mathrm{K}_{\mathrm{v}} 1.2$ expression in the proximal axon became shorter specifically in ventral MSO neurons during fetal development $(29.3 \pm 1.13 \mu \mathrm{m}, n=22$ for E-Pre, $23.9 \pm 1.12 \mu \mathrm{m}, n=21$ for M-Pre, and $19.6 \pm 0.99 \mu \mathrm{m}$, $n=17$ for Term, $p<0.0001$, one-way ANOVA). There was no significant difference in $\mathrm{K}_{\mathrm{v}} 1.2$ expression in dorsal MSO neurons (Figure 5B). Therefore, after hearing onset in M-Pre and Term baboons, $\mathrm{K}_{\mathrm{v}} 1.2$ expression was significantly shorter in ventral MSO neurons rather than dorsal MSO neurons, indicating a tonotopic differentiation of $\mathrm{K}_{\mathrm{v}} 1.2$ channel expression in MSO neurons. In addition, the observed location of the $\mathrm{K}_{\mathrm{v}} 1.2$ channel expression became proximate to the soma of MSO neuron and more superimposed with the immunolabeled with ankyrinG (AnkG), representing the axon initial segment (AIS), in ventral MSO neurons during fetal development (Figure 5C). The length 

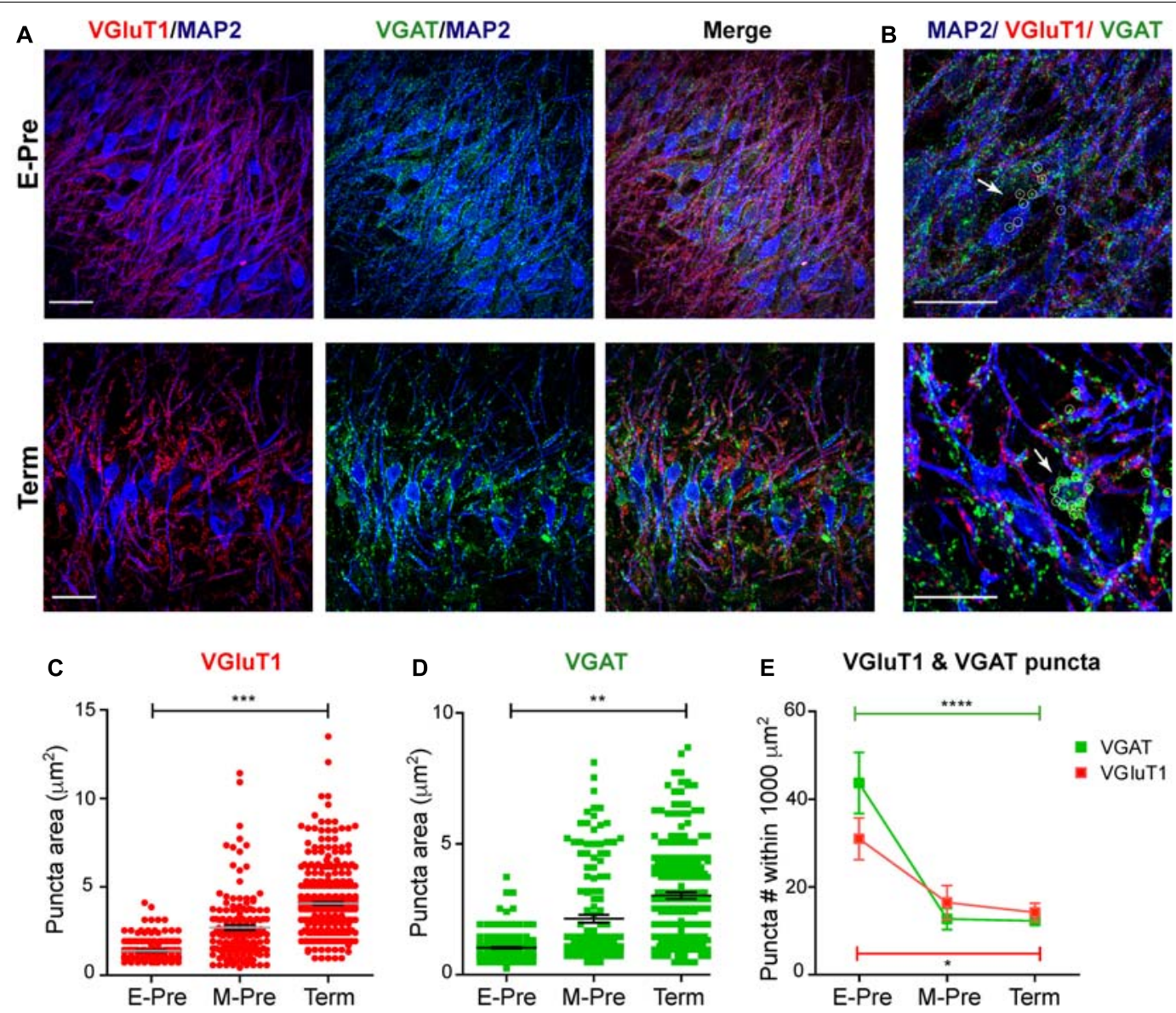

FIGURE 3 | Synaptic inputs on MSO neurons during gestational development of baboon neonates. (A) MSO nuclei were immunostained with MAP2 (blue), VGluT1 (red), and VGAT (green) in the auditory brainstem at E-Pre (top) and Term (bottom). Scale = $50 \mu \mathrm{m}$. (B) Magnified image of MSO neurons. White arrows and small circles indicate VGAT signals (green) on MSO somata. Scale = $50 \mu \mathrm{m}$. (C,D) Changes in the puncta area for VGluT1 (C) and VGAT (D) from E-Pre, M-Pre, and Term neonates. (E) Numbers of VGluT1 and VGAT puncta per a defined area $\left(1000 \mu \mathrm{m}^{2}\right)$ in the MSO. Data were shown as mean \pm s.e.m. ${ }^{\star}<0.05$, ${ }^{\star *}<0.01$, ${ }^{\star * \star}<0.001$, and ${ }^{* \star \star *}<0.0001$, a nested one-way ANOVA.

of AnkG and $\mathrm{K}_{\mathrm{v}} 1.2$ expression were getting shorten during the fetal development, but the location (the distance from soma) only changed in $\mathrm{K}_{\mathrm{v}} 1.2$ expression (Figure 5D). Thus, in E-Pre MSO, $\mathrm{K}_{\mathrm{v}} 1.2$ expression was partially superimposed on AnkG expression and located at more distal axons. In Term MSO, the length of $\mathrm{K}_{\mathrm{v}} 1.2$ expression became shorter and closer to the soma, thus almost co-localized with AnkG.

\section{The AIS of MSO Neurons Undergoes Dynamic Changes After Hearing Onset in the Uterus}

Intrinsic excitability of MSO neurons is important for dictating neuronal response to synaptic inputs and for output signaling. Presynaptic inputs can influence the structural development of axonal domains in the avian auditory brainstem (Kuba et al., 2010). We examined structural changes in the AIS, where voltage-gated $\mathrm{Na}^{+}$channels are clustered, of MSO neurons throughout gestational development. Our previous study in the mouse auditory brainstem found the structure of the AIS in MNTB neurons depends on their functional topographic location along the tonotopic axis, aligning high- to low-frequency sound-responding neurons (HF or LF neurons). Specifically, HF neurons dramatically undergo structural remodeling during early postnatal development (Kim et al., 2019). In M-Pre and Term neonate MSO, AIS length of MSO neurons depended on the topographic location along the dorsal-ventral axis (Figure 6A). Ventral MSO neurons responding to high-frequency sound had a much shorter AIS compared with dorsal MSO neurons in Term baboon neonates $(12.16 \pm 0.876 \mu \mathrm{m}, n=28$ cells in dorsal vs $23.06 \pm 1.007 \mu \mathrm{m}, n=21$ cells in ventral, Figure 6B). During gestational development, the AIS of ventral MSO neurons became significantly shorter $(24.80 \pm 0.422 \mu \mathrm{m}$, $n=61$ cells in 3 E-Pre baboons, $21.61 \pm 0.551 \mu \mathrm{m}, n=40$ cells in $3 \mathrm{M}$-Pre baboons, and $12.16 \pm 0.876 \mu \mathrm{m}, n=28$ cells in 2 Term baboons, $p<0.001$, One-way ANOVA), whereas AIS length of dorsal MSO neurons remained relatively stable (Figure 6B). In the uterus maternal environment including sound experience after hearing onset influences the refinement of the AIS of MSO, which is important to generate action 
A

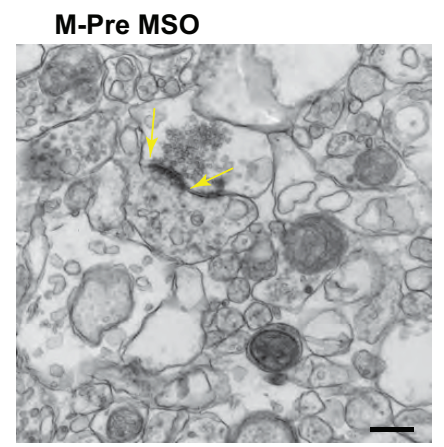

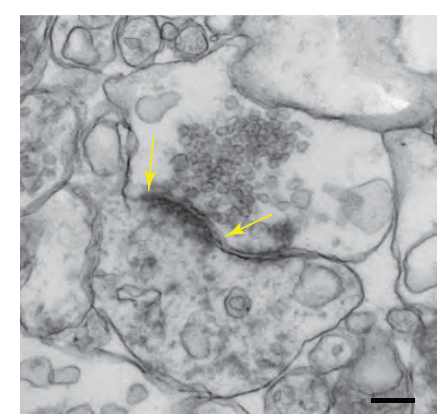

B

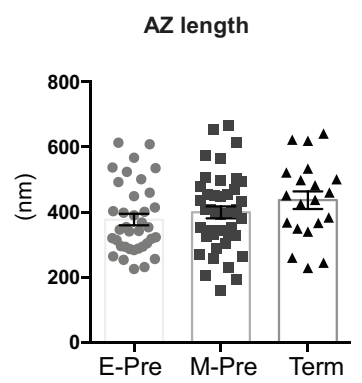

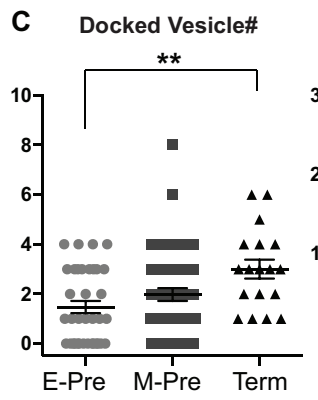
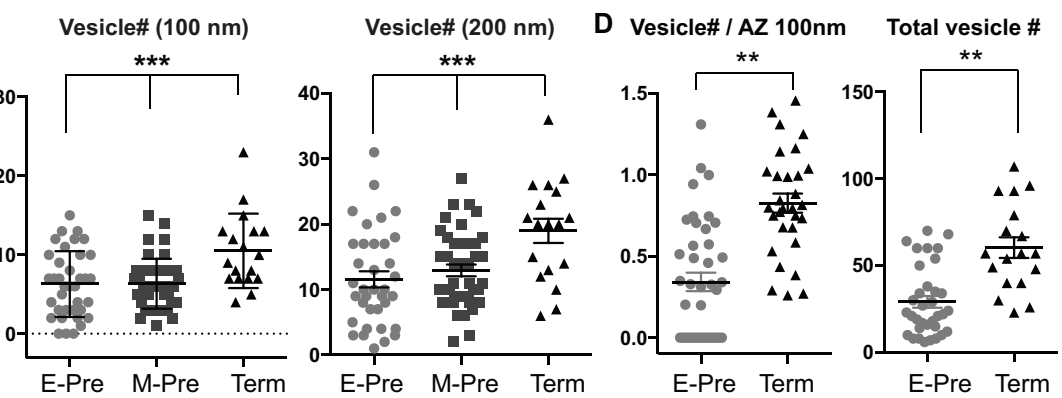

FIGURE 4 | Presynaptic terminal undergoes structural refinement during gestational development. (A) EM images of MSO synapse from M-Pre neonates. Yellow arrows indicate the active zone (AZ) at the presynaptic bouton. Scales $=400$ and $200 \mathrm{~nm}$. (B) AZ length at the presynaptic boutons in the MSO from E-Pre, M-Pre, and Term neonate. (C) The numbers of docked vesicles, which are located within $10 \mathrm{~nm}$ from the AZ, and numbers of vesicles located within 100 and $200 \mathrm{~nm}$ around the AZ in the presynaptic terminal. Data were shown as mean \pm s.e.m. ${ }^{\star \star}<0.01$ and ${ }^{* \star *}<0.001$, one-way ANOVA test. (D) Numbers of docked vesicles in the defined $A Z(100 \mathrm{~nm})$ and numbers of total vesicles in individual boutons. Data were shown as mean \pm s.e.m. ${ }^{\star *}<0.01$, unpaired $t$-test.

potentials and contributes to output signaling of the MSO along the tonotopic axis.

\section{Effect of the NICU's Unnatural Auditory Environment on MSO Development in Preterm Baboons}

Most preterm newborns experience an auditory environment in the NICU, which is markedly different from the uterus (Lahav and Skoe, 2014; Rand and Lahav, 2014). We investigated how shortened time in uterus and altered auditory environment impacts development of morphologic features of MSO neurons in preterm baboon neonates. We compared cellular structures of MSO neurons from baboon neonates with a NICU stay (14 days after birth at 126d GA) and from age-matched baboon neonates delivered at 140d GA (M-Pre). The size of MSO $\left(319,884 \pm 18,137 \mu \mathrm{m}^{2}, n=18\right.$ nuclei from 4 baboons in the NICU) was not significantly different from the age-matched group at $77 \%$ GA $\left(283,045 \pm 29,823 \mu \mathrm{m}^{2}, n=7\right.$ nuclei from 3 M-Pre baboons). There was also no difference in somatic length of MSO neurons, indicating that overall development of MSO was not influenced by the NICU stay. However, NICU experienced preterm baboons displayed the altered AIS length of ventral MSO neurons, thus affecting the tonotopic gradient of AIS length. The AIS of ventral MSO neurons was much longer in neonates with a NICU stay than in the uterus $(23.52 \pm 0.422 \mu \mathrm{m}$, $n=91$ cells in the NICU vs $21.61 \pm 0.551 \mu \mathrm{m}, n=40$ cells in the uterus, $p=0.0013$, unpaired $t$-test). However, there was no difference in dorsal MSO neurons $(26.37 \pm 0.424 \mu \mathrm{m}, n=115$ cells in the NICU vs $25.46 \pm 0.432 \mu \mathrm{m}, n=77$ cells in the uterus, $p=0.1511$, unpaired $t$-test, Figures 7A,B). Therefore, in baboon neonates with auditory experience in the uterus, the MSO showed a clear tonotopic alignment of the AIS (shorter in ventral MSO neurons and longer in dorsal MSO neurons) at 77\% GA, but neonates with a NICU stay did not. This result suggests that shortened time in uterus and limited auditory experience may delay or impair the developmental refinement of the AIS of ventral MSO in the NICU, resulting in an elimination of the tonotopic segregation of the MSO.

In addition to axonal structure of MSO neurons, preterm birth after hearing onset significantly affected the development of synaptic input to the MSO. In age-matched baboon neonates, the VGAT puncta area indicating inhibitory inputs to MSO neurons was significantly reduced in baboon neonates with a NICU stay, compared with neonates in the uterus $\left(1.34 \pm 0.065 \mu \mathrm{m}^{2}, n=157\right.$ puncta from 3 baboons in the NICU vs $2.14 \pm 0.152 \mu \mathrm{m}^{2}, n=154$ puncta in the uterus, $p=0.0088$, unpaired t-test). However, there was no significant difference in VGluT1 puncta area $(2.93 \pm 0.136$ $\mu \mathrm{m}^{2}, \mathrm{n}=158$ puncta in the NICU vs $2.69 \pm 0.142 \mu \mathrm{m}^{2}, n=159$ puncta in the uterus, $p=0.0663$, unpaired $t$-test, Figures 7C,D). These results indicate that shortened time in uterus and NICU stay entailing an unnatural environment may affect both the development of inhibitory input to the MSO and the refinement of axonal structures in MSO development. 
A
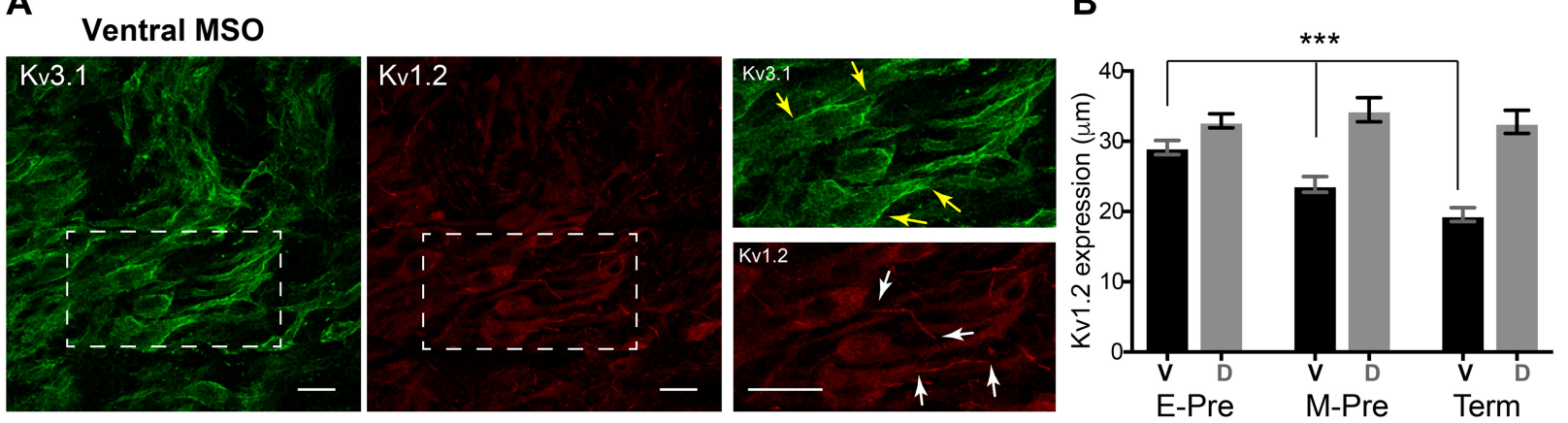

C

\section{Ventral MSO}
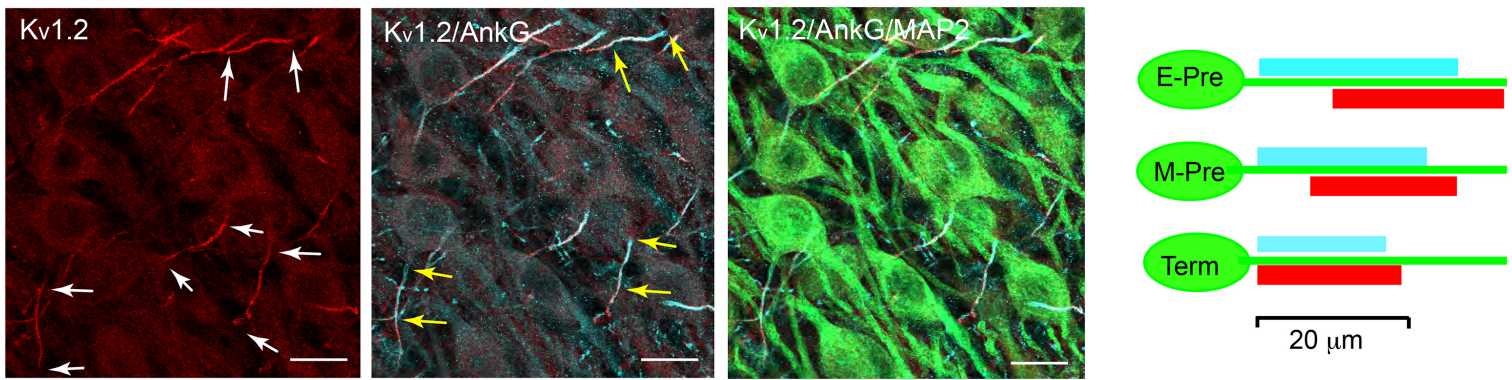

AnkG

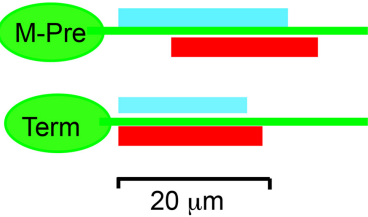

\section{Kv1.2 expression}

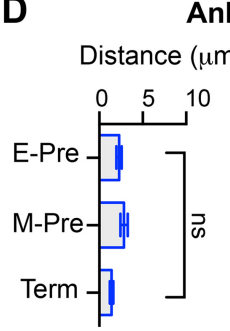

AnkG expression

Length $(\mu \mathrm{m})$
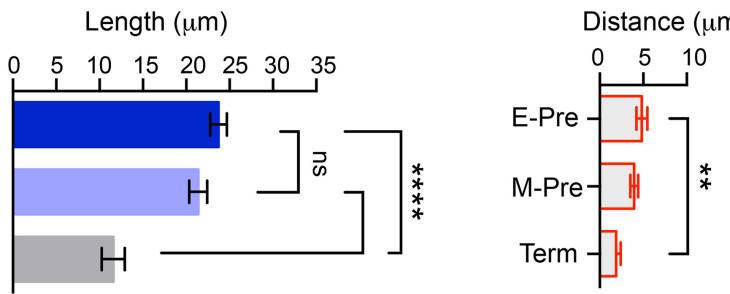

Length $(\mu \mathrm{m})$

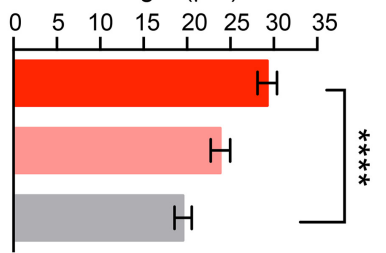

FIGURE 5 | Voltage-activated $\mathrm{K}^{+}$channel expression in the MSO of baboon neonates. (A) Expression of $\mathrm{K}_{\mathrm{v}} 3.1$ (green) and $\mathrm{K}_{\mathrm{v}} 1.2$ (red) channels in the ventral MSO from M-Pre neonate. (Right) Magnified image of MSO neurons immunolabelled by $\mathrm{K}_{\mathrm{v}} 3.1$ and $\mathrm{K}_{\mathrm{v}} 1.2 \mathrm{in}$ boxed area in $\mathrm{A}$. Yellow arrows indicate $\mathrm{K}_{\mathrm{v}} 3.1$ expression on somata and dendrites of MSO neurons and white arrows indicate $K_{v} 1.2$ expression in the axon of MSO. Scale $=20 \mu \mathrm{m}$. (B) Changes in the length of $K_{v} 1.2$ expression on MSO axons in ventral and dorsal MSO in E-Pre, M-Pre, and Term neonates. Data were shown as mean \pm s.e.m. ${ }^{\star \star \star}<0.001$, one-way ANOVA test. (C) $\mathrm{K}_{\mathrm{v}} 1.2$ expression (red) and colocalization with ankyrinG (AnkG, cyan) at the axon initial segment of an MSO neuron (MAP2, green) from an E-Pre baboon neonate. Scale $=20 \mu \mathrm{m}$. (Right) Schematic of $\mathrm{K}_{\mathrm{v}} 1.2$ expression pattern along the proximal axon of MSO neurons during fetal development. (D) The quantitative analysis of AnkG and $\mathrm{K}_{\mathrm{v}} 1.2$ expression (the distance from the soma and the length of expression) along the ventral MSO neurons. ${ }^{* *}<0.01,{ }^{\star \star * *}<0.0001$, and $\mathrm{ns}$ represents "not significant". One-way ANOVA test and post hoc Tukey's multiple comparison test.

\section{DISCUSSION}

The morphologic and physiologic properties of MSO neurons have well been described in rodents such as gerbils and mice (Chirila et al., 2007; Fischl et al., 2016; Nabel et al., 2019). However, most studies using rodents have focused on postnatal development and/or the effect of auditory experience after hearing onset around postnatal day 12 (P12). Here we examined the structural properties of the MSO during fetal development in baboon neonates delivered at different gestational ages and in a NICU. Axonal and synaptic structures of MSO neurons and the overall shape of the MSO nucleus underwent profound refinement in the uterus during the last trimester after hearing onset at around $70 \%$ of GA.
Medial superior olivary nucleus neurons in baboon neonates have a larger soma and more complex primary dendrites as a function of longer gestational ages. In a previous study in gerbils, one or three primary dendrites originated from the soma of MSO neurons had a number of small branches on the distal dendrites. The number of distal dendritic branches per cell decreased over age (from P8 to P15), indicating less complex dendrites (Chirila et al., 2007). Our contradictory findings in baboon neonates could be caused by differences in species or developmental ages. In baboon neonates, MSO nuclei change from a round shape in E-Pre to a thin column shape in Term neonates during the third trimester of gestation, whereas the overall shape and size of MSO nuclei in gerbils do not change based on postnatal ages (Chirila et al., 2007). This could reflect different fetal 
A

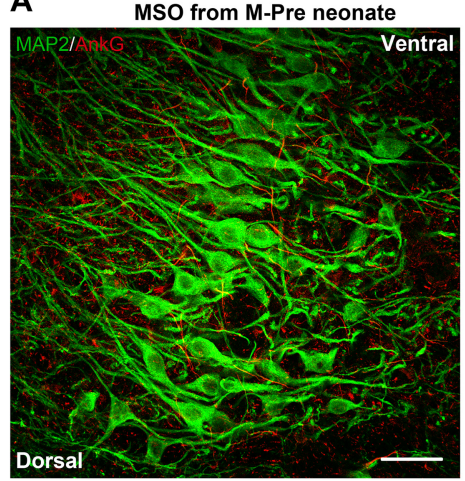

B

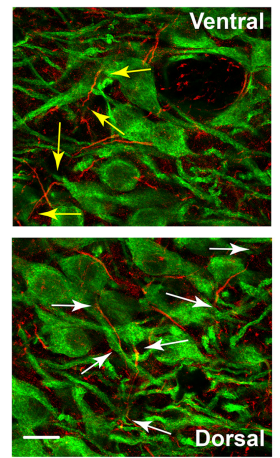

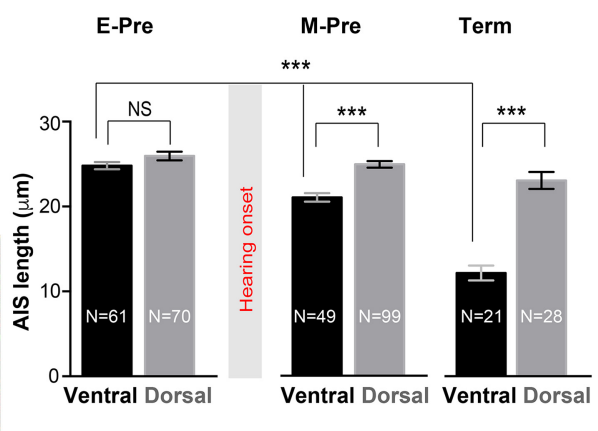

FIGURE 6 | Refinement of the axon initial segment of MSO neurons at different gestational ages of baboon neonates. (A) AnkG and MAP2 expression in ventral MSO from M-Pre neonates. The image includes 50\% position (from the dorsal edge) of the MSO. Magnified images from ventral MSO and dorsal MSO immunostained with MAP2 and AnkG. Arrows indicate the length of AnkG expression. Scale = $50 \mu \mathrm{m}$ (left) and $20 \mu \mathrm{m}$ (right). (B) AIS length (AnkG) in the dorsal MSO and the ventral MSO at different GA. Hearing onset is between 67\% (E-Pre) and 77\% (M-Pre). Data were shown as mean \pm s.e.m. ${ }^{\star \star \star}<0.001$, one-way ANOVA and post hoc Tukey test.

A

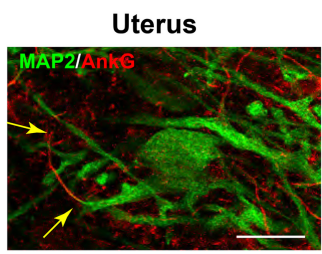

C NICU experienced neonate

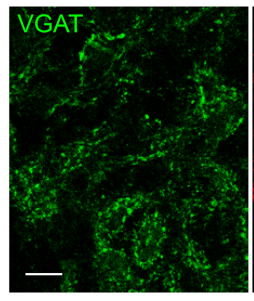

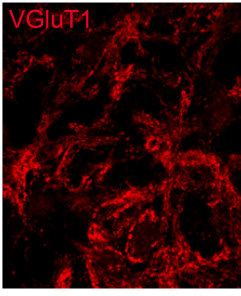

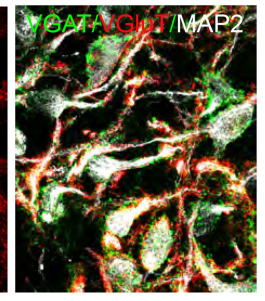

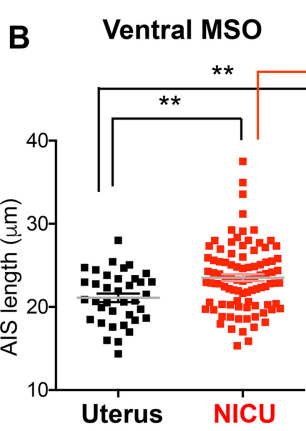

NS

Dorsal MSO

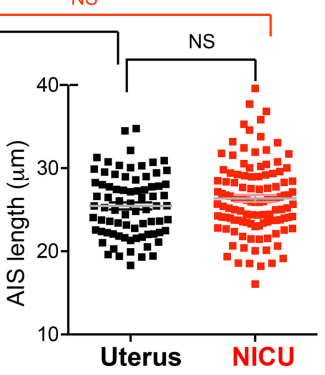

D

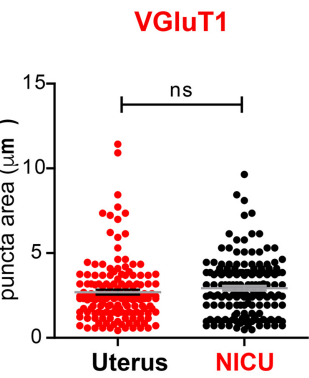

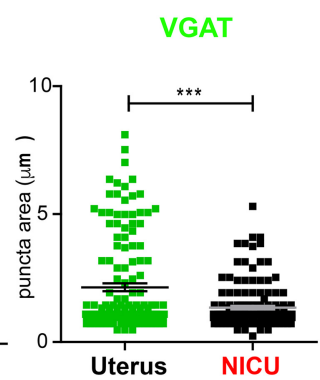

FIGURE 7 | Two-week NICU stay influences structural development of the MSO in baboon neonates. (A) Axon initial segments of MSO neurons were immunolabeled with AnkG (red) and MAP2 in neonates delivered normally at 140d GA (with maternal auditory experience in uterus; Uterus), and age-matched neonates delivered at 126d with a 2-week postnatal stay in the NICU (NICU). Scale $=20 \mu \mathrm{m}$. Arrows indicate the AIS, which was stained with AnkG.

(B) Comparison of AIS length of MSO neurons in the ventral and dorsal MSO in the two groups (Uterus vs NICU). (C) Immunostaining for VGluT1 (red) and VGAT (green) puncta on MSO neurons (MAP2) in neonates with a NICU stay. Scale $=20 \mu \mathrm{m}$. (D) VGluT1 (red) and VGAT (green) puncta area in the MSO from neonates with a NICU stay (NICU) vs in the uterus (Uterus). ${ }^{* *}<0.01$ and ${ }^{* \star *}<0.001$, unpaired $t$-test.

and postnatal developmental effects on the MSO in different species. In addition, our complexity analysis of MSO neurons focused on their somata and primary dendrites, and excluded dendritic branches. In E-Pre neonates, most MSO neurons had distinct bipolar structures with two primary dendrites extending in a medial-lateral orientation. In Term neonates, MSO neurons showed a smaller soma and more primary dendrites stretching in multiple directions. These conflicts between gerbil and baboon neonates could be due to differences in the fetal and postnatal development of the MSO in different species or due to differences in relative developmental ages studied. It should be noted that in this study, morphologic analysis was based on MAP2 immunolabelling for multiple neurons instead of postrecording immunostaining for a single cell. Thus, it is possible 
to underestimate sizes of the distal structures of MSO dendrites located far away from the stomata in baboon neonates.

After hearing onset, spatial refinement of excitatory and inhibitory synaptic inputs on the dendritic and somatic surface is critical for MSO neurons to allow synaptic integration for fine-tuning of the coincidence mechanisms (Kapfer et al., 2002; Grothe and Pecka, 2014). Similar to the MSO in gerbils (Kapfer et al., 2002), the distribution of inhibitory inputs in MSO was refined after hearing onset in baboons, in the uterus and throughout fetal development (Kim et al., 2014). Before hearing onset in E-Pre neonates, both VGluT1 and VGAT signals were detected at the somata and dendrites without a distinguishable pattern. After hearing onset, VGAT signals, indicating inhibitory inputs, were concentrated on the alignment of MSO somata throughout Term neonates, whereas VGluT1 signals were concentrated on dendrites. Our results in baboon neonates support the concept that auditory experience in the uterus promotes spatial refinement of excitatory and inhibitory inputs on MSO neurons, allowing them to detect interaural time differences in low-frequency hearing mammals (Kapfer et al., 2002). In addition to the spatial distribution of synaptic inputs, the VGluT1 and VGAT puncta on MSO neurons significantly enlarged after hearing onset during the last trimester in the pregnancy. Our ultrastructural analysis using TEM supports the idea that the increased size of VGluT1 and VGAT puncta may be due to increased synaptic vesicle numbers around the active zone in the individual boutons. Although inhibitory and excitatory synapses were not distinguishable in these analyses, most synapses had a similar staining pattern during fetal development. The enlargement of synapses with spatial orientation observed in the structural analysis could contribute to increase the efficacy of binaural computation during fetal development in a non-human primate MSO (Reyes et al., 1996; Kapfer et al., 2002).

Medial superior olivary nucleus neurons play a role in detecting coincidences within interaural time differences in the range of a few tens of microseconds for computing sound location. To achieve this task with remarkable acuity, voltageactivated $\mathrm{K}^{+}\left(\mathrm{K}_{\mathrm{V}}\right)$ channels critically contribute to setting a short integration time constant of the membrane and a typical spiking pattern (Scott, 2005; Chirila et al., 2007; Fischl et al., 2016; Nabel et al., 2019). In baboon neonates, a single spike of MSO neurons in response to a long-depolarizing current injection displayed a relatively high threshold and a lower amplitude, similar to findings in rodents. In gerbils and mice, the amplitude of spikes and input resistance in MSO neurons progressively decreased after hearing onset (Scott, 2005; Fischl et al., 2016), whereas we observed no significant difference in baboons between E-Pre and M-Pre neonates. In gerbils, low voltage-activated potassium $\left(\mathrm{K}_{\mathrm{V}} 1\right)$ channels and high voltageactivated $\left(\mathrm{K}_{\mathrm{v}} 3\right)$ channels were detected along the MSO neuron from soma to dendrites (Nabel et al., 2019). In mice, $K_{v} 1$ currents determine the AP threshold and firing pattern and $\mathrm{K}_{\mathrm{v}} 3$ currents contribute to AP width (Fischl et al., 2016). In baboons, we detected $\mathrm{K}_{\mathrm{v}} 3.1$ channel along the MSO neurons from the soma to the primary dendrites, whereas $\mathrm{K}_{\mathrm{v}} 1.2$ channels appear to be restricted to the axon initial segment of MSO neurons. The lengths of $\mathrm{K}_{\mathrm{V}} 1.2$ expression progressively reduced along the axon initial segment of MSO neurons during fetal development. In rodents, $\mathrm{K}_{\mathrm{v}} 1$ currents were increased during postnatal development; this could drive developmental changes in intrinsic properties of MSO neurons, resulting in a lower amplitude and a higher threshold of APs (Scott, 2005; Fischl et al., 2016). We observed no significant changes in AP waveforms in MSO neurons between E-Pre and M-Pre neonates (Figure 1D). Future studies will be required to elucidate whether a shorter length of $\mathrm{K}_{\mathrm{v}} 1.2$ expression in baboon MSO is directly associated with reduced $\mathrm{K}_{\mathrm{v}} 1$ currents and/or changes in AP shape during fetal development including Term neonates.

Preterm infants admitted to the NICU frequently show some abnormalities in auditory brainstem responses (Jiang et al., 2012). The transduction of sound information along the auditory pathway affects activity-dependent circuitry formation during a critical period for auditory development, i.e., when the nervous system is especially sensitive to certain environmental sound stimuli (Friauf and Lohmann, 1999). Preterm newborns experience a drastic change from the maternal environment of the uterus to the NICU. The missing of maternal sound inputs (e.g., heart-beating sound in the uterus) in the NICU coincided with a significantly delayed or impaired synaptic and axonal development of MSO neurons that normally occurs during the critical period in baboon neonates. Our findings support that shortened time in uterus and altered environment in the NICU critically impacts the structural refinement of neurons and synapses in the MSO that occurs during perinatal development. The NICU generally has a noisy environment with relatively high-frequency sound ( $>500 \mathrm{~Hz}$ broad spectrum and 70-75 dB in daytime), which derived from routine hospital noise levels (Lahav and Skoe, 2014). Thus, future studies need to evaluate how either the noisy environment in the NICU or maternal sound deprivation separately or synergistically contributes to the anatomic and functional development of the MSO in baboon neonates.

\section{MATERIALS AND METHODS}

\section{Animals}

We used 15 baboon brains, including 3 term and 8 preterm baboons (either sex), and 4 preterm baboons (delivered at 126 days of gestational ages and kept in the NICU for postnatal 14 days) obtained from the laboratory of Dr. Cynthia L. Blanco (Department of Pediatrics, University of Texas Health Science Center San Antonio [UTHSCSA]). Baboons were used in accordance with approved UTHSCSA Institutional Animal Care and Use Committee protocols. Preterm baboons were delivered at the Texas Biomedical Research Institute in San Antonio. Preterm baboon care has been described in detail (Blanco et al., 2013; Kim et al., 2014). Briefly, term baboons were delivered naturally (at 180 d GA), whereas preterm baboons were delivered via cesarean section at $125 \pm 2 \mathrm{~d}$ GA ( $67 \%$ of GA) and at $140 \pm 2 \mathrm{~d} \mathrm{GA}$ (77\% of GA). Preterm baboon neonates were intubated immediately after birth and chronically ventilated for a planned survival of $14 \mathrm{~d}$ in the NICU (with sound attenuation, $<40 \mathrm{~dB}$, in isolated room). Central intravenous lines were placed shortly after birth for fluid 
management and parenteral nutrition. In the NICU, preterm baboons were treated with corticosteroids, antibiotics, ketamine, vitamin $\mathrm{K}$, and blood transfusions (Blanco et al., 2013; Kim et al., 2014). Any possible effects of extreme preterm birth independent from NICU experience were not assessed in this study, as NICU interventions were necessary for the survival of the neonates.

\section{Slice Preparation}

Brainstem slices were prepared from term and preterm baboon brainstems after necropsy. Brainstems were immersed in icecold low-calcium artificial CSF (aCSF) containing the following (in $\mathrm{mM}$ ): $125 \mathrm{NaCl}, 2.5 \mathrm{KCl}, 3 \mathrm{MgCl}_{2}, 0.1 \mathrm{CaCl}_{2}, 25$ glucose, $25 \mathrm{NaHCO}_{3}, 1.25 \mathrm{NaH}_{2} \mathrm{PO}_{4}, 0.4$ ascorbic acid, 3 myo-inositol, and 2 Na-pyruvate, bubbled with carbogen $\left(95 \% \mathrm{O}_{2}, 5 \%\right.$ $\mathrm{CO}_{2} ; \mathrm{pH}=7.3-7.4$; osmolality of 310-320 mOsm). Transverse brainstem slices (200 $\mu \mathrm{m}$ thick) were prepared using a microtome (VT1200S, Leica). After cutting, the slices were transferred to an incubation chamber containing normal aCSF bubbled with carbogen and maintained at $35^{\circ} \mathrm{C}$ for $30 \mathrm{~min}$ and thereafter at room temperature. Normal aCSF was the same as the slicing aCSF, but with $1 \mathrm{mM} \mathrm{MgCl}$ and $2 \mathrm{mM} \mathrm{CaCl}_{2}$.

\section{Electrophysiology}

Whole-cell patch-clamp recordings were performed in normal aCSF at room temperature $\left(22-24^{\circ} \mathrm{C}\right)$. The pipette solution contained (in $\mathrm{mM}$ ): $130 \mathrm{~K}$-gluconate, $20 \mathrm{KCl}, 5 \mathrm{Na}_{2}$ phosphocreatine, 10 HEPES, 5 EGTA, $4 \mathrm{Mg}$-ATP, and $0.3 \mathrm{GTP}$, $\mathrm{pH}$ adjusted to 7.3 with $\mathrm{KOH}$. EPSCs or action potentials (APs) were recorded in normal aCSF using the voltage or currentclamp mode of the EPC-10 (HEKA Electronik, Lambrecht/Pfalz, Germany). Patch electrodes had resistances of 2.5-3 $\mathrm{M} \Omega$ and the initial uncompensated series resistance (Rs) was $<20 \mathrm{M} \Omega$. Recordings were not corrected for the predicted liquid junction potential of $11 \mathrm{mV}$. APs were elicited by current injection in current-clamp mode. Data were filtered at $2.9 \mathrm{kHz}$ and digitized at a $10-50 \mu$ s sampling intervals.

\section{Immunohistochemistry}

After electrophysiologic recordings, brainstem slices (150$200 \mu \mathrm{m}$ ) were fixed with 4\% (wt/vol) paraformaldehyde in phosphate buffer saline (PBS) solution for $30 \mathrm{~min}$. Free-floating sections were blocked in $4 \%$ goat serum and $0.3 \%$ Triton X-100 in PBS for $1 \mathrm{~h}$. Primary antibodies were guinea pig polyclonal antivesicular glutamate transporter 1 (VGluT1, 1:3000, Chemicon), anti-vesicular GABA transporter (VGAT, 1:1000, Synaptic Systems Ca\# 131-002), anti-microtubule associated protein 2 (MAP2, 1:200, Millipore), mouse monoclonal anti-ankyrinG (AnkG; 1:100; UC Davis/NIH NeuroMab Facility Cat\# 75-146, RRID:AB_10673030), mouse monoclonal anti-K 1.2 (1:250; UC Davis/NIH NeuroMab Facility Cat\# 75-008, RRID:AB_2296313), mouse monoclonal anti-K 3.1 (1:250; UC Davis/NIH NeuroMab Facility Cat\# 73-041). After 3 washes with PBS containing $0.1 \%$ Tween 20, slices were incubated with different Alexa488 goat anti-mouse IgG1 or 568 goat anti-mouse IgG2b or 568 goat anti-rabbit or 647 goat anti-guinea pig secondary antibodies (1:1000; Invitrogen) accordingly for $2 \mathrm{~h}$ at room temperature. Slices were then rinsed with PBS containing $0.1 \%$
Tween 20 and were cover- slipped using mounting medium (Vectashield; Vector Laboratories). Stained slices were viewed on a confocal laser-scanning microscope (Carl Zeiss LSM-710) at 488,568 , and $633 \mathrm{~nm}$ using a pinhole of $1 \mathrm{AU}$ and $20 \mathrm{x}$ (0.8 NA), 40x (oil-immersion, $1.30 \mathrm{NA}$ ) objectives. Z-stack images were acquired at a digital size of $1024 \times 1024$ pixels with optical section separation ( $z$ interval) of $0.5 \mu \mathrm{m}$. Images were imported into Fiji (Image J) (Schindelin et al., 2012), ZEN (Carl Zeiss), and CellProfiler for analyses. To analyze MSO area, we utilized "draw spline contour tool" in the ZEN or Fiji to outline the MSO, defined by the boundary of the bipolar extension of the MAP2 + neuron cluster. In VGluT1 and VGAT puncta, regions of interest were selected based on MAP2 staining and overlapped with VGluT1 and VGAT staining using CellProfiler. The numbers and sizes of puncta were analyzed using the analysis modules "IdentifyPrimaryObjects and IdentifySecondaryObjects" with settings of (1) typical diameter of objects in pixel units (Min., Max.) = 3, 10; (2) method to distinguish clumped objects = intensity; (3) discard objects outside the diameter range = yes; (4) discard objects touching the border of the image = yes; and (5) threshold $=0.2 \sim 0.3$. For AIS, $2 \mathrm{D}$ compressed Z-stack images were analyzed using the Fiji program as we have described previously (Kim et al., 2019). Briefly, the AIS was determined as the proximal axon where the fluorescence intensity of ankyrin G staining was $>10 \%$ of the peak signal. Length of the AIS was measured by the segmental line profile in the Fiji program.

\section{Theoretical Fractal Dimension Analysis}

The complexity of dendritic arbors was examined by determining fractal dimensions (Karperien et al., 2013). The box-counting method was applied for fractal analysis using fractal3e software ${ }^{1}$. The number $(\mathrm{N})$ of squares (boxes) in a square grid contacted by the dendrites was counted, as grids with decreasing sizes of squares are placed over the cell. The fractal dimension is depicted as the relationship between increasing $\mathrm{N}$ and decreasing size of squares, with larger numbers indicating increased complexity (Chirila et al., 2007).

\section{Transmission Electron Microscopy}

Brains were removed and $400-\mu$ m-thick samples of brainstem MSO area were dissected out followed by primary fixation in $1 \%$ glutaraldehyde/4\% paraformaldehyde. Further processing was performed by the UTHSCSA Electron Microscopy Lab. Briefly, each brainstem was post-fixed with $1 \%$ Zetterqvist's buffered osmium tetroxide, dehydrated, and embedded in PolyBed resin at $80^{\circ} \mathrm{C}$ in an oven. Tissue containing the MSO was cut into 90$\mathrm{nm}$ ultrathin sections and placed on copper grids. The sections were then stained with uranyl acetate and Reynold's lead citrate. The samples were imaged on a JEOL 1400 electron microscope using Advanced Microscopy Techniques software. A total of 20-40 synapses was analyzed from 3 baboons for each age group.

\footnotetext{
${ }^{1}$ http://cse.naro.affrc.go.jp/sasaki/fractal/fractal-e.html
} 
The number of docked vesicles per the active zone was measured for each synapse at a final magnification of $80,000 \times$. The active zone was defined as the dark presynaptic density contacting the postsynaptic density. Docked and clustered vesicles were defined as those within $10 \mathrm{~nm}$ and $200 \mathrm{~nm}$ of the presynaptic active zone, respectively (Satzler et al., 2002).

\section{Analysis and Statistics}

Electrophysiologic data were analyzed and presented using Igor Pro (Wavemetrics, OR). All statistical analyses were performed in Prism (GraphPad Software). Normality of datasets was analyzed using the D'Agostino and Pearson's omnibus test. Parametric or non-parametric tests were carried out accordingly. $\alpha$ values were set to 0.05 , and all comparisons were two-tailed. To compare two groups, unpaired t-tests or Mann-Whitney U tests were carried out. For three or more groups, a nested one-way ANOVA with post hoc Tukey's multiple comparison test was used. Significance was set at $p<0.05$. Data were shown as the mean \pm standard error of the mean (s.e.m.) with $n$ values representing the number of animals per experimental group or the number of neurons per group where indicated. In box and whisker plot, boxes indicate $25-75 \%$ interquartile range and horizontal lines in boxes indicate median. Whiskers show 5\% $\sim 95 \%$ range and dots show outliers that reside outside the whisker range. Differences were deemed statistically significant when $p$-values were $<0.05$.

\section{REFERENCES}

Agrawal, S., Rao, S. C., Bulsara, M. K., and Patole, S. K. (2018). Prevalence of autism spectrum disorder in preterm infants: a meta-analysis. Pediatrics 142:e20180134. doi: 10.1542/peds.2018-0134

Blanco, C. L., McGill-Vargas, L. L., McCurnin, D., and Quinn, A. R. (2013). Hyperglycemia increases the risk of death in extremely preterm baboons. Pediatr. Res. 73, 337-343. doi: 10.1038/pr.2012.184

Chirila, F. V., Rowland, K. C., Thompson, J. M., and Spirou, G. A. (2007). Development of gerbil medial superior olive: integration of temporally delayed excitation and inhibition at physiological temperature. J. Physiol. 584, 167-190. doi: 10.1113/jphysiol.2007.137976

Deitch, J. S., and Rubel, E. W. (1984). Afferent influences on brain stem auditory nuclei of the chicken: time course and specificity of dendritic atrophy following deafferentation. J. Comp. Neurol. 229, 66-79. doi: 10.1002/cne.902290106

Edwards, D. A., Henderson-Smart, D. J., Pettigrew, A. G., Wetzlar, A., and Phippard, A. F. (1994). Brainstem auditory evoked response development in preterm and term baboons (Papio hamadryas). Brain Res. Dev. Brain Res. 82, 181-184. doi: 10.1016/0165-3806(94)90161-9

Fanaroff, A. A., Stoll, B. J., Wright, L. L., Carlo, W. A., Ehrenkranz, R. A., Stark, A. R., et al. (2007). Trends in neonatal morbidity and mortality for very low birthweight infants. Am. J. Obstet. Gynecol. 196, 147.e1-8.

Fischl, M. J., Burger, R. M., Schmidt-Pauly, M., Alexandrova, O., Sinclair, J. L., Grothe, B., et al. (2016). Physiology and anatomy of neurons in the Medial Superior Olive (MSO) of the mouse. J. Neurophysiol. 116, 2676-2688. doi: 10.1152/jn.00523.2016

Franken, T. P., Bremen, P., and Joris, P. X. (2014). Coincidence detection in the medial superior olive: mechanistic implications of an analysis of input spiking patterns. Front. Neural Circuits 8:42. doi: 10.3389/fncir.2014.00042

Friauf, E., and Lohmann, C. (1999). Development of auditory brainstem circuitry. activity-dependent and activity-independent processes. Cell Tissue Res. 297, 187-195. doi: 10.1007/s004410051346

\section{DATA AVAILABILITY STATEMENT}

The original contributions presented in the study are included in the article/supplementary material, further inquiries can be directed to the corresponding author/s.

\section{ETHICS STATEMENT}

The animal study was reviewed and approved by UTHSCSA Institutional Animal Care and Use Committee.

\section{AUTHOR CONTRIBUTIONS}

$\mathrm{EK}, \mathrm{KN}, \mathrm{CB}$, and $\mathrm{JK}$ performed the experiments and data analyses. JK wrote the manuscript, supervised the project, and revised the manuscript. All authors reviewed and approved the final manuscript.

\section{FUNDING}

This work was supported by a grant from the National Institute on Deafness and Other Communication Disorders (NIDCD, R01 DC03157), a Southwest National Primate Research Center (SNPRC) Pilot Grant, and a Children's Health Pilot Program Grant (UTHSCSA), all to JK.

Gallo, J., Dias, K. Z., Pereira, L. D., Azevedo, M. F., and Sousa, E. C. (2011). [Auditory processing evaluation in children born preterm]. J. Soc. Bras. Fonoaudiol. 23, 95-101.

Griffith, J. L., Shimony, J. S., Cousins, S. A., Rees, S. E., McCurnin, D. C., Inder, T. E., et al. (2012). MR imaging correlates of white-matter pathology in a preterm baboon model. Pediatr. Res. 71, 185-191. doi: 10.1038/pr.2011.33

Grothe, B., and Pecka, M. (2014). The natural history of sound localization in mammals-a story of neuronal inhibition. Front. Neural Circuits 8:116. doi: 10.3389/fncir.2014.00116

Jiang, Z. D., Chen, C., and Wilkinson, A. R. (2012). Brainstem auditory response findings in term neonates in intensive care unit. J. Matern. Fetal Neonatal Med. 25, 2746-2749. doi: 10.3109/14767058.2012.718385

Johnson, S., Ring, W., Anderson, P., and Marlow, N. (2005). Randomised trial of parental support for families with very preterm children: outcome at 5 years. Arch. Dis. Child 90, 909-915. doi: 10.1136/adc.2004.057620

Joris, P. X., Smith, P. H., and Yin, T. C. (1998). Coincidence detection in the auditory system: 50 years after Jeffress. Neuron 21, 1235-1238. doi: 10.1016/ s0896-6273(00)80643-1

Kapfer, C., Seidl, A. H., Schweizer, H., and Grothe, B. (2002). Experiencedependent refinement of inhibitory inputs to auditory coincidence-detector neurons. Nat. Neurosci. 5, 247-253. doi: 10.1038/nn810

Karperien, A., Ahammer, H., and Jelinek, H. F. (2013). Quantitating the subtleties of microglial morphology with fractal analysis. Front. Cell. Neurosci. 7:3. doi: 10.3389/fncel.2013.00003

Khurana, S., Remme, M. W., Rinzel, J., and Golding, N. L. (2011). Dynamic interaction of Ih and IK-LVA during trains of synaptic potentials in principal neurons of the medial superior olive. J. Neurosci. 31, 8936-8947. doi: 10.1523/ jneurosci.1079-11.2011

Kim, E. J., Feng, C., Santamaria, F., and Kim, J. H. (2019). Impact of auditory experience on the structural plasticity of the ais in the mouse brainstem throughout the lifespan. Front. Cell Neurosci. 13:456. doi: 10.3389/fncel.2019. 00456 
Kim, S. E., Lee, S. Y., Blanco, C. L., and Kim, J. H. (2014). Developmental profiles of the intrinsic properties and synaptic function of auditory neurons in preterm and term baboon neonates. J. Neurosci. 34, 11399-11404. doi: 10.1523/jneurosci.4734-13.2014

Kuba, H., Oichi, Y., and Ohmori, H. (2010). Presynaptic activity regulates $\mathrm{Na}(+)$ channel distribution at the axon initial segment. Nature 465, 1075-1078. doi: 10.1038 /nature09087

Kulesza, R. J., and Grothe, B. (2015). Yes, there is a medial nucleus of the trapezoid body in humans. Front. Neuroanat. 9:35. doi: 10.3389/fnana.2015.00035

Kulesza, R. J., and Mangunay, K. (2008). Morphological features of the medial superior olive in autism. Brain Res. 1200, 132-137. doi: 10.1016/j.brainres.2008. 01.009

Lahav, A., and Skoe, E. (2014). An acoustic gap between the NICU and womb: a potential risk for compromised neuroplasticity of the auditory system in preterm infants. Front. Neurosci. 8:381. doi: 10.3389/fnins.2014.00381

Lieu, J. E., Tye-Murray, N., Karzon, R. K., and Piccirillo, J. F. (2010). Unilateral hearing loss is associated with worse speech-language scores in children. Pediatrics 125, e1348-e1355.

McClure, M. M., Peiffer, A. M., Rosen, G. D., and Fitch, R. H. (2005). Auditory processing deficits in rats with neonatal hypoxic-ischemic injury. Int. J. Dev. Neurosci. 23, 351-362. doi: 10.1016/j.ijdevneu.2004.12.008

McMahon, E., Wintermark, P., and Lahav, A. (2012). Auditory brain development in premature infants: the importance of early experience. Ann. N. Y. Acad. Sci. 1252, 17-24. doi: 10.1111/j.1749-6632.2012.06445.x

Miller, D. J., Lackey, E. P., Hackett, T. A., and Kaas, J. H. (2013). Development of myelination and cholinergic innervation in the central auditory system of a prosimian primate (Otolemur garnetti). J. Comp. Neurol. 521, 3804-3816. doi: $10.1002 / \mathrm{cne} .23379$

Moore, J. K. (2002). Maturation of human auditory cortex: implications for speech perception. Ann. Otol. Rhinol. Laryngol. Suppl. 189, 7-10. doi: 10.1177/ 00034894021110s502

Moore, J. K., and Linthicum, F. H. Jr. (2007). The human auditory system: a timeline of development. Int. J. Audiol. 46, 460-478. doi: 10.1080/ 14992020701383019

Moster, D., Lie, R. T., and Markestad, T. (2008). Long-term medical and social consequences of preterm birth. N. Engl. J. Med. 359, 262-273. doi: 10.1056/ nejmoa0706475
Nabel, A. L., Callan, A. R., Gleiss, S. A., Kladisios, N., Leibold, C., Felmy, F., et al. (2019). Distinct distribution patterns of potassium channel sub-units in somato-dendritic compartments of neurons of the medial superior olive. Front. Cell. Neurosci. 13:38. doi: 10.3389/fncel.2019. 00038

O'Connor, K. (2012). Auditory processing in autism spectrum disorder: a review. Neurosci. Biobehav. Rev. 36, 836-854.

Rand, K., and Lahav, A. (2014). Impact of the NICU environment on language deprivation in preterm infants. Acta Paediatr. 103, 243-248.

Reyes, A. D., Rubel, E. W., and Spain, W. J. (1996). In vitro analysis of optimal stimuli for phase-locking and time-delayed modulation of firing in avian nucleus laminaris neurons. J. Neurosci. 16, 993-1007.

Satzler, K., Söhl, L. F., Bollmann, J. H., Borst, J. G., Frotscher, M., Sakmann, B., et al. (2002). Three-dimensional reconstruction of a calyx of Held and its postsynaptic principal neuron in the medial nucleus of the trapezoid body. J. Neurosci. 22, 10567-10579.

Schindelin, J., Arganda-Carreras, I., Frise, E., Kaynig, V., Longair, M., Pietzsch, T., et al. (2012). Fiji: an open-source platform for biological-image analysis. Nat. Methods 9, 676-682.

Scott, L. L. (2005). Posthearing developmental refinement of temporal processing in principal neurons of the medial superior olive. J. Neurosci. 25, 7887-7895.

Wilson-Costello, D., Friedman, H., Minich, N., Fanaroff, A. A., and Hack, M. (2005). Improved survival rates with increased neurodevelopmental disability for extremely low birth weight infants in the 1990s. Pediatrics 115, 997-1003.

Conflict of Interest: The authors declare that the research was conducted in the absence of any commercial or financial relationships that could be construed as a potential conflict of interest.

Copyright (c) 2021 Kim, Nip, Blanco and Kim. This is an open-access article distributed under the terms of the Creative Commons Attribution License (CC BY). The use, distribution or reproduction in other forums is permitted, provided the original author(s) and the copyright owner(s) are credited and that the original publication in this journal is cited, in accordance with accepted academic practice. No use, distribution or reproduction is permitted which does not comply with these terms. 